\title{
LOW REYNOLDS NUMBER MICRO-FLUIDIC DEVICES
}

\author{
James P. Brody and Paul Yager \\ Center for Bioengineering, University of Washington \\ Seattle, WA 98109-2141
}

\begin{abstract}
The fluid dynamics in microfabricated fluidic devices is inherently in the low Reynolds number regime. By recognizing this, efficient micro-devices can be designed. Two such devices are described, a diffusion-based filter and a virtual valve. The diffusion-based filter differentially separates particles and molecules based on diffusion coefficient while diluting their concentration. Results and a simple analysis indicate that the dilution of the particles is exponentially related to the diffusion coefficient. The virtual valve functions as a switch, directing flow at the intersection of three channels by manipulating the pressure driving the flow. Switching times as fast as $100 \mathrm{~ms}$ are demonstrated. These devices are unique to the micro-scale-they would not work as well if scaled up.
\end{abstract}

\section{INTRODUCTION}

It has recently become possible to fabricate intricate fluid handling systems with channel sizes as small as $1 \mu \mathrm{m}$ using microfabrication techniques. These devices can be mass produced inexpensively and some may soon be in widespread use for simple analytical tests $[1,2]$. There are many advantages these miniaturized devices could offer, including the promise of performing some analytical functions more rapidly and on smaller samples than with macroscopic systems.

However, miniaturization of analytic instruments is not a simple matter of reducing their size. At small scales different effects become important, rendering some processes inefficient and others useless. It may also be difficult to replicate smaller versions of some devices because of material or process limitations. For these reasons it may prove necessary to develop new methods for performing common laboratory tasks on the micro-scale.

This paper presents two individual micro-devices which function as the equivalent of macroscopic-scale filters and valves, but which operate on entirely different principles. These devices would be inefficient on the macroscopic scale, but function quite well on the microscopic scale.

These devices are able to function because of several important physical principles. Typical micro-fluidic devices operate in the low Reynolds number regime where turbulent mixing and inertia are absent. Mixing can only be done by diffusion,j and diffusion of small molecules occurs rapidly over distances of $1-10 \mu \mathrm{m}$.

\section{FLUID DYNAMICS}

Microfabricated fluid systems often operate under low Reynolds number conditions. The Navier-Stokes equations governing fluid flow can be written as

$$
-\nabla P+\eta \nabla^{2} u=\rho\left(\frac{\partial u}{\partial t}+(u \cdot \nabla) u\right)
$$

where $P$ is the applied pressure, $\mathbf{u}$ is the velocity vector, $\rho$ is the fluid density and $\eta$ is the viscosity of the fluid. The left side of this equation represents the forces on the fluid due to applied pressure and viscosity and the right hand side represents "inertial forces". A useful quantity is the ratio of the inertial forces to the viscous force. The magnitude of this ratio,

$$
R=\frac{\rho\left(\frac{\partial u}{\partial t}+(u \cdot \nabla) u\right)}{\eta \nabla^{2} u}=\frac{\rho r^{2}}{\eta \tau}+\frac{\rho u r}{\eta},
$$

is written in terms of the characteristic size (typically the radius) of the channel, $r$, and the time scale over which the velocity is changing, $\tau$ ( where $u / \tau=\partial u / \partial t$.)

Fluid flow behavior in the steady state, $\tau \rightarrow \infty$, is characterized by the Reynolds number, $R_{e}=\rho u r / \eta$. Because of the small sizes and slow velocities, microfabricated fluid systems are often in the low Reynolds number regime $\left(R_{e} \ll 1\right)$. In this regime, inertial effects, which cause turbulence and secondary flows, are negligible; viscous effects dominate the dynamics. (See [3] for a good introduction to low Reynolds number fluid dynamics.) Because of the absence of inertial effects, at low Reynolds number all mixing is done by diffusion.

\section{DIFFUSION}

Diffusion of small molecules occurs radiply over typical microfabricated dimensions. The relationship between the size of a particle, $R_{0}$, the diffusion coefficient, $D$, and temperature, $T$, is due to Einstein and for the simplest case, spherical particles, this can be written as[4],

$$
D=\frac{k_{b} T}{6 \pi \eta R_{\circ}} .
$$

The characteristic distance, $l$, which a particle with diffusion coefficient $D$ will diffuse in time, $t$, is $l=\sqrt{D t}$. Table 1 gives some typical diffusion coefficients and characteristic times.

Table 1: Some typical values for different sized particles and molecules. The characteristic time to diffuse $10 \mu \mathrm{m}$ is given.

\begin{tabular}{ccc}
\hline \hline Panicle & \multicolumn{1}{c}{$\overline{D\left(20^{\circ} \mathrm{C}\right)}$} & $t$ \\
\hline $0.5 \mu \mathrm{m}$ sphere & $5 \times 10^{-9} \mathrm{~cm}^{2} / \mathrm{sec}$ & $200 \mathrm{sec}$ \\
Protein (hemoglobin) & $7 \times 10^{-7} \mathrm{~cm}^{2} / \mathrm{sec}$ & $1 \mathrm{sec}$ \\
Small Molecule (fluorescein) & $5 \times 10^{-6} \mathrm{~cm}^{2} / \mathrm{sec}$ & $0.2 \mathrm{sec}$ \\
\hline
\end{tabular}

\section{SILICON PROCESSING}

Silicon micromachining was done in the Washington Technology Center microfabrication facility. The process was modified from previous work [5] to avoid the problem of coating deep features uniformly with photoresist. In our experience, spin coating photoresist on a wafer that had features greater than a few microns deep led to a non-uniform coating that either left undeveloped areas after exposure or areas uncoated with photoresist. These problems were avoided with this process.

This process required two mask levels. One level defined connecrion ports, which were etched completely through the wafer, and the second level defined the fluid transport channels on the face of the wafer, typically only $10 \mu \mathrm{m}$ deep. 


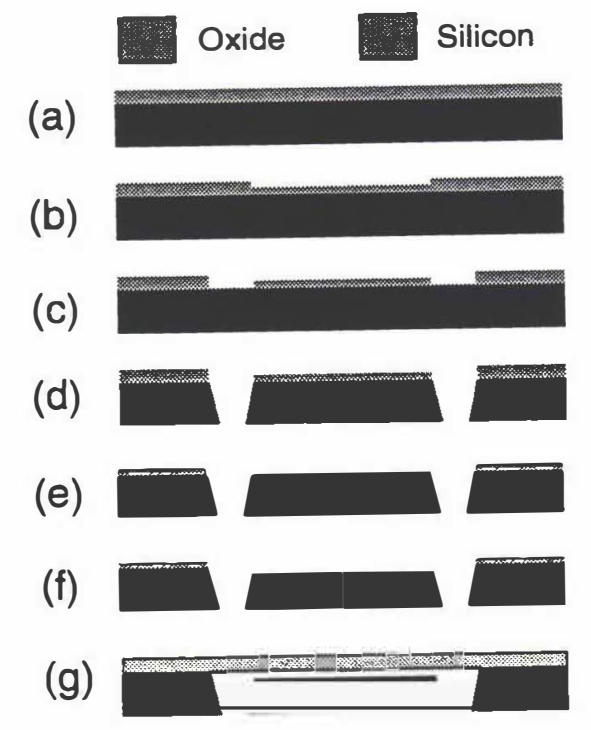

Figure 1: A schematic diagram of the silicon processing. (a) A $1 \mu \mathrm{m}$ thick wet thermal oxide is grown on a 3" silicon wafer. (b) This oxide is photolithographically patterned with the flow channels and etched to a depth of $600 \mathrm{~nm}$. (c) The wafer is recoated with photoresist and patterned with the through-hole connections. This oxide is completely removed from this pattern. (d) EDP etching is done to etch completely through the wafer (approximately $400 \mu \mathrm{m}$ ). (e) An oxide etch is performed to uniformly remove $400 \mathrm{~nm}$ of oxide from the wafer. (f) The flow channels are etched into the silicon approximately $10 \mu \mathrm{m}$ deep. (g) The wafer is anodically bonded to a 3" disk of Pyrex glass.

We started with 4" chrome masks made to our specifications by Photo Sciences, Inc. (Torrance, CA) and 3" silicon wafers (100, ntype) with $1000 \mathrm{~nm}$ of wet thermal $\mathrm{SiO}_{2}$ grown on them. A primer (P-10M, MicroSi, Phoenix, AZ) was spun on at 3000 rpm. About one micron of AZ-1512 (Hoechst) photoresist was deposited by spin coating (3000 rpm), and this was followed by a soft bake (30 min at $90 \mathrm{C}$ ).

A contact aligner was used to align and expose wafers. Exposure time (nominally 15 seconds) was varied to yield best results. No post-exposure bake was done. Wafers were developed in AZ-351 (Hoechst) (diluted 4:1) for one minute, and rinsed in DI water. Blue tack tape (Semiconductor Equipment Corporation, Moorpark, CA) was applied to the backsides of the wafers to protect the oxide from the oxide etch.

The fluid channel mask was exposed first. After developing, the wafers were immersed in a buffered oxide etch (BOE, 10:1 HF (49\%) and $\mathrm{NH}_{4} \mathrm{~F}(10 \%)$ ) to etch away $600 \mathrm{~nm}$ of the unprotected oxide. The blue tack tape was removed by hand, and the photoresist was removed in an acetone rinse.

Wafers were again coated with photoresist and the fluid connection mask was aligned and exposed on the wafers. These wafers were developed and blue tack tape was once again applied to the backsides of the wafers to protect the oxide, and then immersed in the BOE to remove all the unprotected oxide. Blue tack tape and photoresist were removed as before.

Silicon etching was done in a mixture of ethylene-diamine, pyrocatechol, and water (EDP F-etch) set up in a reflux boiling flask. This etch attacks the $\{100\}$ planes of silicon at a rate of about $100 \mu \mathrm{m}$ per hour[6]. First fluid connection ports were etched through the wafer, then $400 \mathrm{~nm}$ of the oxide were removed in a BOE. Finally, flow channels between fluid connection ports were etched about $10 \mu \mathrm{m}$ deep. After final processing the wafers were cleaned in a Piranha bath and rinsed in DI water.

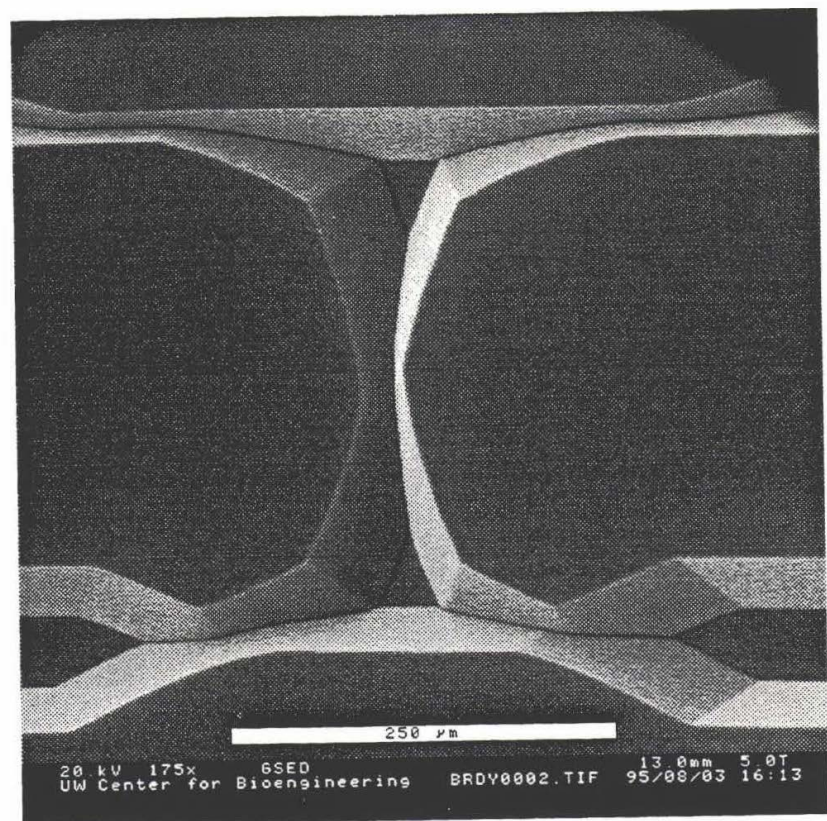

Figure 2: A scanning electron micro graph showing the central portion of a diffusion-based filtration device.

We used anodic bonding [7] to attach Pyrex glass to the silicon devices. We obtained 3" diameter pieces of Pyrex glass (100 $\mu \mathrm{m}$ thickness) from Esco Products Inc (Oak Ridge, New Jersey). First, the silicon and Pyrex glass were immersed in a solution of $\mathrm{H}_{2} \mathrm{O}_{2}, \mathrm{NH}_{4} \mathrm{OH}$, and $\mathrm{H}_{2} \mathrm{O}(1: 4: 6)$ heated to $50 \mathrm{C}$. This process removes any organic matter on the surfaces and also makes the surfaces hydrophilic. After 20 minutes in this solution, the silicon and Pyrex were placed in a rinser-dryer. Upon removing, the Pyrex glass was immediately placed into contact with the silicon wafer and set on a hot plate for anodic bonding. Bonding was done at $485 \mathrm{C}$ with $800 \mathrm{~V}$ applied between the Pyrex and the silicon.

The Pyrex-silicon structures were diced into individual $1 \mathrm{~cm}$ by $1 \mathrm{~cm}$ devices by manually marking the silicon with a diamond tipped scribe and gently applying stress along the scratch. The Pyrex-silicon fractured along the silicon fault without problems.

\section{EXPERIMENTAL SETUP}

Flow visualization was done by flowing dilute suspensions of fluorescent micro-spheres through the device. We added one drop of $0.5 \mu \mathrm{m}$ diameter fluorescent micro-spheres (1\% solids, from Duke Scientific, Palo Alto, CA) to $5 \mathrm{ml}$ of DI water.

This mixture was introduced into the device, and observations were made using a Zeiss ICM-405 inverted microscope equipped with an XF34 fluorescent set ( $535 \mathrm{~nm}$ excitation, $590 \mathrm{~nm}$ emission) from Omega Optical (Burlington VT). We observed the fluorescence of the spheres using both a silicon intensified target camera (SIT-66x, from Dage-MTI) and a Pulnix monochrome CCD video camera. We recorded the observations on video tape. Some images were also digitized using a frame grabber (Data Translation or Power Macintosh 7100AV). 


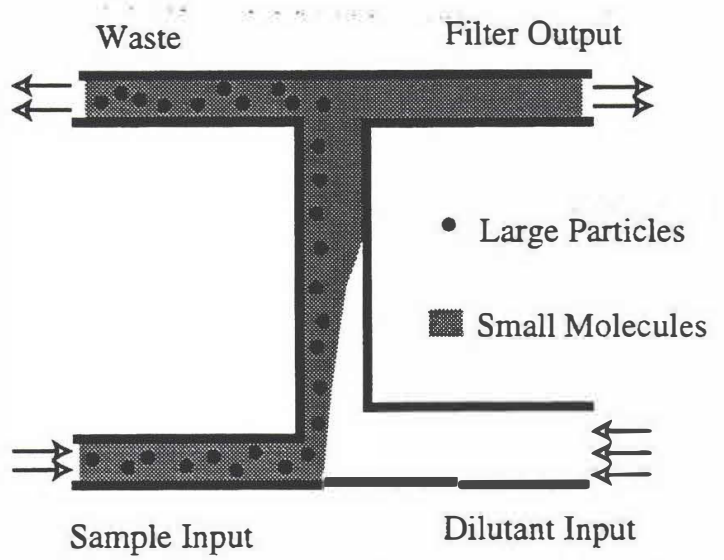

Figure 3: The concept of diffusion-based filtration. Two flow streams are brought into contact. Because the flow is at low Reynolds number conditions, no mixing occurs at the intersection. As the two flow streams move down the central channel, some molecules diffuse across. At the end of the central channel, the flow is again split (not necessarily at the same place) into two streams. The output is preferentially enriched with smaller molecules over larger ones.

\section{DIFFUSION BASED SEPARATION}

The concept of diffusion-based separation is described in Figure 3. The process relies on the non-mixing nature of low Reynolds number flow along with the fast diffusion times for small molecules. The idea was originally implemented in a system with two glass plates separated by a shim by Giddings[8]. We present here a greatly modified version of this implemented in a microfabricated fluid system.

A detailed analysis will be published elsewhere, but the main result is that a molecule (with diffusion coefficient, $D$ ) present in the input stream at a concentration $C_{o}$ will be present in the output stream at a concentration $C$,

$$
C \sim C_{\circ} \exp \left(-D_{f} / D\right)
$$

where the parameter, $D_{f}$, quantifies the device, $D_{f}=\lambda_{b}^{2} / t_{R}$. The time a particle spends in the central channel is $t_{R}$, and the shortest distance a particle must diffuse to go from the input stream to the output stream is $\lambda_{b}$ (the distance between the inlet splitting plane and the outlet splitting plane in [8].) The resolution of the separation is set by the minimum concentration that can be detected.

The pressures at the sample input port and at the dilutant port were set by filling a tube which fed the ports to the appropriate height with liquid. Typically a pressure difference of about $2 \mathrm{~cm}$ of water gave a maximum velocity in the central channel of about $100 \mu \mathrm{m} / \mathrm{sec}$. The waste and filter output ports were held constant. Measurements were made with two different devices. (with different central channel lengths) and with varying flow rates to vary the parameters $\lambda_{b}$ and $t_{R}$ and hence $D_{f}$. The data roughly follow the predicted behavior.

\section{VIRTUAL VALVES}

Virtual valves have been demonstrated in electrokinetically driven flow[9], but apparently not in pressure driven flow. The same principles apply in each case-by controlling the pressure (as opposed to the voltage for electrokinetics) at each port of a " $T$ " shaped device, the flow can be directed one way or another at the intersection of channels.
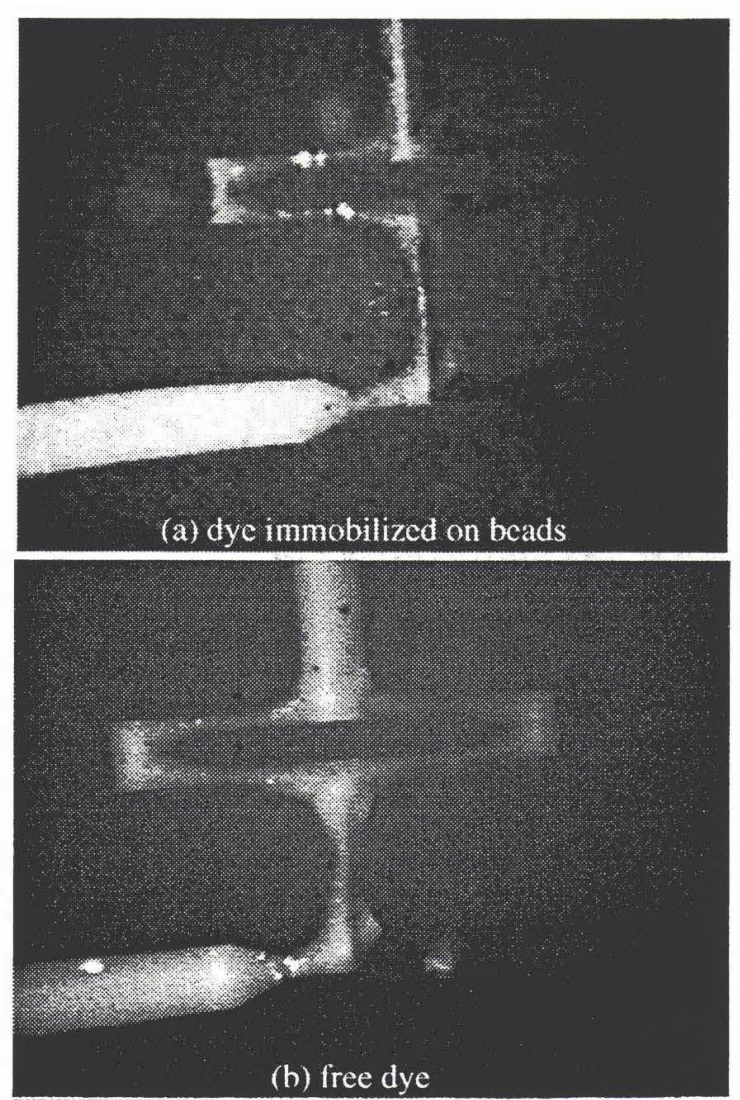

Figure 4: Digitized video images from the diffusion-based filter. As in Figure 3, flow is from the lower left and right up through the central channel and splits into the waste (upper left) and output (upper right). In (a) the fluorescent dye is immobilized on $0.5 \mu \mathrm{m}$ spheres which diffuse too slowly to end up in the output stream in any significant concentration. In (b), the dye (fluorescein), is free to diffuse, and it readily diffuses throughout the central channel. In this device the output and waste channel are tied together to simplify the pressure control.

Output Concentration vs Diffusion Coefficient

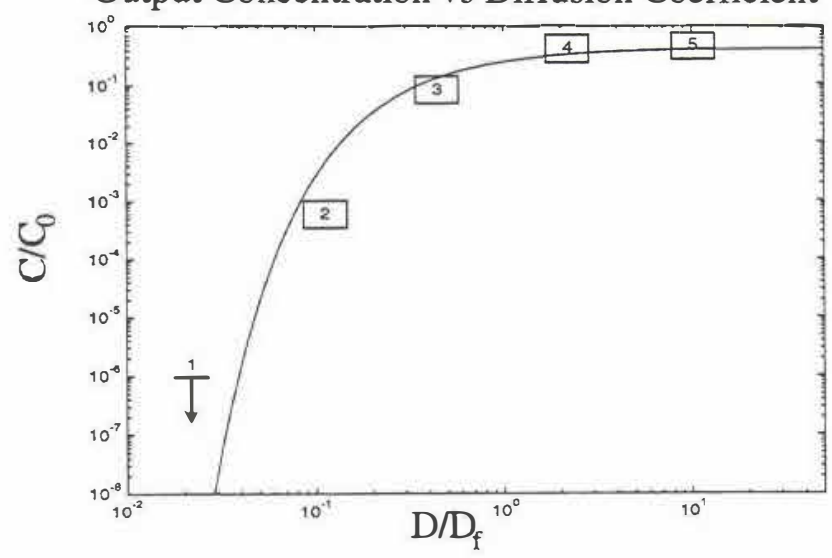

Figure 5: Data from the diffusion-based filter. The ratio of concentration in the output to concentration in the input $\left(C / C_{0}\right)$ is plotted versus the diffusion coefficient of the molecule of interest $(D)$. (The diffusion coefficient is measured in terms of the device parameter, $D_{f}$, which is a function of flow rate, central channel length, and diffusion distance, see text.)) Concentrations were measured by either counting fluorescent spheres or measuring fluorescence intensity of fluorescein with a photo diode. The solid line is the expected behavior from equation (4). Errors are or order the sizes of the boxes. 
(a)

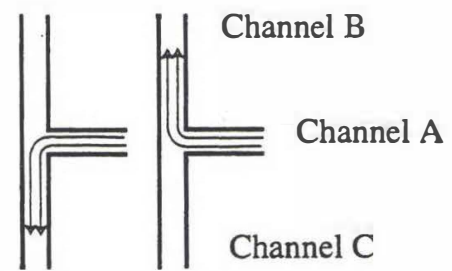

Flow before switching.
Flow after switching.

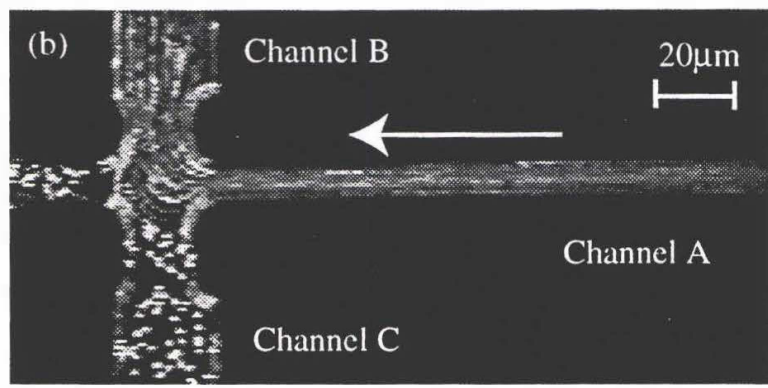

Figure 6: A virtual valve. (a) The flow at the intersection of three channels can be directed by controlling the pressures behind Channels $A, B$, and $C$. (b) A fluorescent picture of the virtual valve in action. Flow is from right to left in channel $A$ and up channel $B$. Note that the fluid is stationary in channel $C$.

The fastest rate at which one can expect to switch the flow without significant mixing is given by the requirement that $R<1$ from equation (2). Faster switching may be possible with careful control of the geometry, but as long as $R<1$ the geometry is irrelevant. Tabie 2 gives some typical examples for water in different sized channels.

Table 2: Approximate fastest switching allowed while still maintaining low Reynolds number conditions. (Time given for water in different channel diameters.

\begin{tabular}{cc}
\hline Diameter of Channel & Switching time \\
\hline $10 \mu \mathrm{m}$ & $100 \mu \mathrm{s}$ \\
$100 \mu \mathrm{m}$ & $10 \mathrm{~ms}$ \\
$1 \mathrm{~mm}$ & $1 \mathrm{~s}$ \\
\hline
\end{tabular}

These experiments were performed by driving the fluid through $10 \mu \mathrm{m}$ deep channels with a pressure of about 1 PSI over a total length of $1 \mathrm{~cm}$. The pressure was provided from a compressed nitrogen cylinder. A small pressure regulator was built which allowed control over pressure at two of the ports (the third was allowed to float at atmospheric pressure.) A valve was installed between the pressure regulator and the device which allowed rapid switching between the ports. For instance as in figure $6 \mathrm{a}$, before switching the relative pressures were Channel A, 1 PSI; Channel B, 0.5 PSI; Channel C, 0 PSI. After switching, they were Channel A, 1 PSI; Channel B, O PSI; Channel C, 0.5 PSI

\section{CONCLUSIONS}

Micro-fluidic devices operate in the low Reynolds number regime. The fluid dynamics in this regime is dominated by viscous forces, rather than inertial ones. Because of this, micro-fluidic devices are inherently different than macroscopic ones. By understanding the fluid dynamics and diffusion processes which occur at the micro-scale, efficient and novel devices can be designed to perform common laboratory functions needed in any lab-on-a-chip.

Switching Time

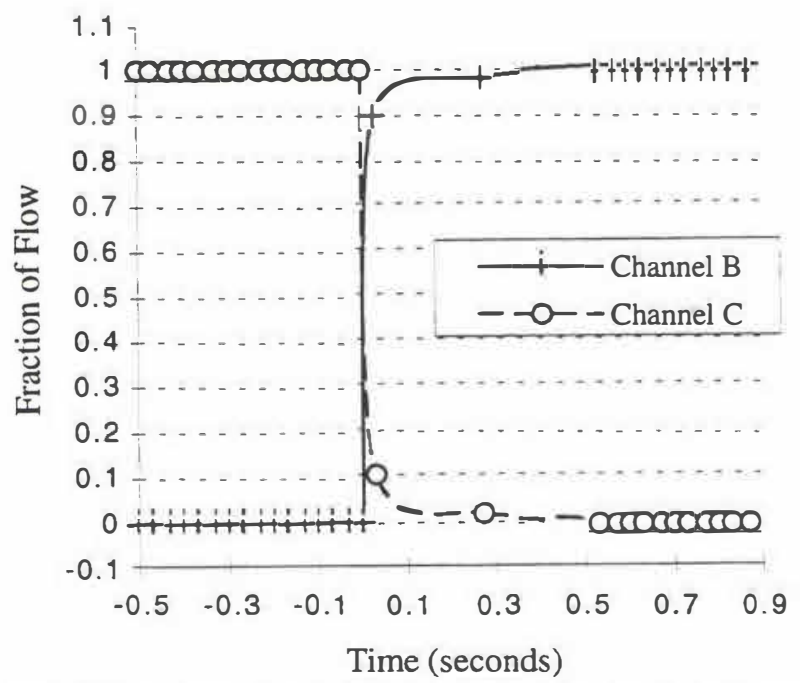

Figure 7: Data from the switching of the virtual valve. Video images were analyzed frame-by-frame and the relative flow rates in two channels were recorded. The switching is completely done in $\sim 100 \mathrm{~ms}$. An analysis indicates that this could be done as quickly as $100 \mu \mathrm{s}$ in a $10 \mu \mathrm{m}$ deep channel. In our experiment, the switching time is limited by our pressure control system.

\section{ACKNOWLEDGMENTS}

We thank H. Sho Fuji and D. Schutte for expert advice on microfabrication and $\mathrm{M}$. Holl for help with the pressure control system. This work was supported by the Washington Technology Center, ARPA (grant \# DAMD17-94-J-4460) and Senmed Medical Ventures, Inc.

\section{REFERENCES}

[1] J. Michael Ramsey, Stephen C. Jacobson, and Michael R. Knapp. Microfabricated chemical measurement systems. Nature Medicine, 1(10):1093-1096, 1995.

[2] D. Jed Harrison, Karl Fluri, Kurt Seiler, Zhonghui Fan, Carlos S. Effenhauser, and Andreas Manz. Micromachining a miniaturized capillary electrophoresis-based chemical analysis system on a chip. Science, 261:895-897, August 1993.

[3] E. M. Purcell. Life at low Reynolds number. American Journal of Physics, 45(1):3-11, 1977.

[4] E. L. Cussler. Diffusion: Mass Transfer in Fluid Systems. Cambridge University Press, 1984

[5] James P Brody, Thor Osborn, Fred K Forster, and Paul Yager. A planar microfabricated fluid filter. In Transducers '95, 1995.

[6] Kurt E. Petersen. Silicon as a mechanical material. Proceedings of the IEEE, 70(5):420-457, 1982.

[7] George Wallis and Daniel I. Pomerantz. Field assisted glass-metal sealing. Journal of Applied Physics, 40:3946-3949, 1969.

[8] Shulamit Levin and J Calvin Giddings. Continuous separation of particles from macromolecules in split-flow thin (SPLITT) cells. Journal of Chemical Technology in Biotechnology, 50:43$56,1991$.

[9] Andreas Manz, Carlo S Effenhauser, Norbert Burggraf, D Jed Harrison, Kurt Seiler, and Karl Fluri. Electroosmotic pumping and electrophoretic separations for miniaturized chemical analysis systems. J. Micromech. Microeng, 4:257-265, 1994. 